\title{
Increase in nonspecific resistance of catfish (Clarias gariepinus) in industrial aquaculture
}

\author{
E. M. Romanova*, V. V. Romanov, V. N. Lyubomirova, L. A. Shadieva, and T. M. Shlenkina
}

Ulyanovsk State Agrarian University named after P.A. Stolypin, Ulyanovsk 432017, Russia

\begin{abstract}
The article discusses prospects for use of the theory of adaptogens in demand in medicine and in the fish breeding practice. The obtained results show that the adaptogens use at the early stages of catfish ontogenesis (Clarias Gariepinus, Burchell 1822) can cause the condition of nonspecific increased resistance and expand the adaptive flexibility range of this specie. Adaptogen trekrezan used in our experiments (Oxyethylammonium methylphenoxiacetate) is a synthetic analogue of the natural ginseng. Its application in the embryo culture process has shown positive results. It has reduced the level of the developmental anomaly, increased the first stage larvae hatching and their viability, significantly decreased the mortality rate and reduced the level of cannibalism common for catfish larvae. We have determined that the larvae breeding in the trekrezan medium has considerably increased their growth rate, and weight gain. The obtained results are new, and the adaptogens use at the early stages of ontogenesis in industrial catfish aquaculture is recommended. The Russian Foundation for Basic Research has supported our study with the grant No. 18-416-730005.
\end{abstract}

\section{Introduction}

Catfish is a prospective object of high-tech industrial aquaculture. This type of fish has a high growth rate. It is hardy, adapted to high-density holding and has an epibranchial organ. This organ allows breathing the ambient oxygen. Catfish are known to have delicious meat and we recommend it for dietary and child nutrition. [1,2].

Currently, catfish is a well-known object of fish breeding in Asia, Africa, Europe and America [1]. Since 1994, Russia has introduced the catfish into industrial aquaculture but they are still not widespread. The climatic conditions in most regions of Russia do not allow catfish breeding in natural reservoirs; we use the recirculating aquaculture system to grow them.

This thermophile species requires warm rooms in the winter season. The optimum temperature for catfish growing is $26{ }^{0} \mathrm{C}$. At this temperature, catfish can increase their weight up to $1.5 \mathrm{~kg}$ or more within six months.

Other species are unable to show such a result. Currently, in the technologies of fast growing, the genetic potential of catfish productivity has not been fully discovered.

In aquaculture, catfish loses the ability of natural reproduction. In industrial aquaculture, the growing of this species is possible only through hormonal stimulation [3-6].

In aquaculture, the fundamental knowledge of ontogenesis, ecology, regulation of physiological processes, hormonal regulation of ovogenesis, cyclicism of reproductive function, adaptive flexibility of pituitaryovarian mechanism, norms and pathology of germinal cells is necessary for complete genetic potential fulfillment with regards to catfish productivity. Such knowledge is not enough yet.

Catfish are known to have a multiple spawning and can lay fish eggs several times a year. Caviar of this type is small, has a rich dark green color. It has a high nutritional value, a good taste and can be a highlydemanded food product.

In industrial aquaculture, the fish eggs maturation periodicity of Clarias Gariepinus has not been studied, there is not enough knowledge about the factors determining its qualitative and quantitative characteristics. In nature, seasonal reproduction is typical for catfish. In the natural habitat, the gonadotropic hormone level in the pituitary organs has seasonal distribution in this species. In winter, the hormonal level is decreased by 4-6 times in comparison to the summer season. We have not identified if the breeding in an artificial environment that mitigates the effect of natural factors has the same regularity.

The constant tension of the reproductive system is known to affect the quality and number of males and females germinal cells and accordingly the quality and quantity of the produced fish eggs and fries. The studies on catfish have not been conducted; they have been carried out on sturgeon, which does not belong to a species capable of multiple spawning within the season.

Gametogenesis, the qualitative and quantitative characteristics of female spawn under conditions of

*Corresponding author: vvr-emr@yandex.ru 
intensive functioning of the reproductive system in sturgeons have significantly changed and deteriorated.

The adaptation failure that is characterized by the loss of fertilizing capacity in germinal cells due to reproductive system depletion can occur in both fish and mammals.

There is no research in this field currently available. Perhaps for this reason there is still no catfish caviar production, although these fish species have the repeated spawning and this idea can be successful.

This work is devoted to the search for possible ways to expand the adaptive flexibility of catfish breeding in high-tech aquaculture. We used adaptogens for this purpose.

Adaptogens are preparations of a plant, chemical and biological nature, creating in the organism "a state of nonspecific increased resistance" - SNIR [6, 7].

We know that the organism is protected from physical and chemical damage and viral diseases in the state of SNIR caused by the adaptogens administration. The decrypted molecular mechanisms of adaptogens action on human and animal organisms are in strong demand in medicine and other fields. [8]. Adaptogens increase immunity, increase hardiness, optimize the major functions of the organism systems, have antioxidant properties, stimulate regeneration processes; ensure disease prevention and extend length of their life $[9,10]$.

Adaptogen trekrezan is a universal adaptogen with a broad-spectrum action that we recommend for use in animal husbandry.

Trekrezan increases the absorption of methionine, which is involved in hormones formation in the anterior pituitary gland, prevents growth retardation, regulates lipid metabolism.

We have studied the effectiveness of the trekrezan use to increase the resistance and expansion of catfish adaptive flexibility at the early stages of ontogenesis in high-tech industrial aquaculture.

\section{Materials and method}

We have conducted our research in the Laboratory of experimental biology and aquaculture at the Ulyanovsk State Agrarian University. The object of the study was mature females and males of catfish with average weight from 1.2 to $1.7 \mathrm{~kg}$.

To stimulate artificial spawning we have applied surfagon (synthetic nanopeptide, analog of gonadotropin-releasing hormone luliberin, $10 \mathrm{mg} / \mathrm{ml}$ ) in an amount of $1.5 \mathrm{ml}$ per $\mathrm{kg}$ in fish weight. It is the inductor of ovogenesis and spermatogenesis, sulphered (Sulpiride) was used as the effect intensifier. Surfagon is a synthetic equivalent of gonadotropin-releasing hormone-luliberin, which binds to the receptors of the cells in the anterior lobe of the hypophysis, stimulating the hypophysis gonadotropic function - luteinizing hormone and follicle stimulating hormone. It causes a short-term increase in the level of sex hormones in the blood inducing the maturation of caviar and sperm.
The fertilized eggs flowed from the female abdomen in 11-12 hours after hormonal stimulation. The stage of the caviar maturity was determined cytologically. It is possible to distinguish the mature caviar morphologically, at maturation of gonads oocytes are transparent, follow one by one and not stick together in a bolus.

Maturation of the gonads in male catfish can be determined only by invasive method because in the artificial breeding their sperms do not flow spontaneously. The full or limited resection of the testis is carried out to release the mature sperm. After the operation, we have stitched the abdomen of males with a surgical suture. Catfish, as we have shown, have a high regenerative ability [6] and can be used several times in reproduction after checking the offspring quality. The received testicles were dispergated to obtain sperm.

Artificial fertilization of fish eggs was carried out without the water use, dripping the sperm of males on it and then mixing it quickly and accurately. Right after mixing we treated the fertilized eggs with an aqueous solution of the adaptogen that activated the fertilization process. The fish eggs were being fertilizing and swelling with mixing for several minutes. Then we have washed the fertilized eggs from the remaining sperm with an aqueous solution of trekrezan in a dose of $1.0 \mathrm{mg} / \mathrm{l}$ for 20 minutes. Then fish eggs samples were taken, and the number of fertilized oocytes was estimated.

The incubation of fertilized eggs of catfish was carried out on the metal nets at the water temperature $28^{\circ} \mathrm{C}$ and the oxygen content not less than $90 \%$; we added the adaptogen into the incubation medium in a dose of $1.0 \mathrm{mg} / \mathrm{l}$, water flow rate was $3 \mathrm{l} / \mathrm{min}$. The yolk fry hatching began in a full day and lasted for several hours. The part of the hatched yolk fry was calculated from the number of dead embryos remaining on the net.

On the 3rd day after hatching, the yolk sac resolved in the larvae, they switched to active feeding and turned into the second stage larvae. Over the next 30 days, we observed the growth and development of larvae introducing the adaptogen into the aquariums water and starter feeds. At this phase, we studied the weight, height, developmental anomalies, and larvae survival. We weighed larvae at the beginning and the end of the experiment. In the experimental and control groups, the net and average daily gain was calculated by the obtained data.

The same procedures as in the experimental group were carried out in the control group but without the adaptogen use. For other parameters, the control group did not differ from the experimental one.

The application efficiency of adaptogen trekrezan was estimated by fertilization rate of oocytes ( $\%)$, by hatching of yolk fry, by the number of anomalies in the development of the embryos ( \%), by the larvae survival, by weight gain. The experiments were carried out in triplicate.

Statistical analysis of the data available from experiments and observations was carried out with standard biometrics. 
Determination of the mean value was conducted using the following formula:

$$
M=\frac{\sum_{n}^{1} x_{i}}{n}
$$

where $M$ - mean value;

$X_{i}$ - variant values;

$n$ - the number of observations.

The formula for the standard deviation was:

$$
\delta=\left[\frac{\left(M-x_{i}\right)^{2}}{n-1}\right]^{1 / 2} \text {. }
$$

The error of the mean value $\mathrm{m}$ was calculated using the formula:

$$
m=\frac{\delta}{n-1} .
$$

We used Student's test to determine the statistical significance of the differences in the mean values of the two samples. The expression was used to calculate the criterion

$$
t=\frac{\left(M_{1}-M_{2}\right)}{\sqrt{\left(m_{1}^{2}-m_{2}^{2}\right)}} .
$$

We compared the calculated value of test with the table value criterion at the chosen level of confidence probability $\mathrm{p} \leq 0.05$ and the number of degrees of freedom i. They consider the differences statistically unreliable if the calculated value of the criterion is $t_{p}<t_{s t}$.

\section{Results}

At the first research stage, we have assessed the impact of the adaptogen on fertilization of eggs. In the experimental group with the presence of trekrezana, the fertilization index was $86.33 \pm 4.6 \%$, and it was higher than in the control group, where it was $65.67 \pm 4.96 \%$. The figure 1 shows the results.

In the culture of fertilized eggs in the norm, some part of the embryos dies. In our experiments, the highest embryos mortality occurred in the control group. In this group, the hatching of the first stage larvae was low and amounted to $41.0 \pm 5.34 \%$. In the experimental group, this index was significantly higher $-71.67 \pm 5.03 \%$. The figure 1 represents the results.

The increasing viability of embryos and the hatching of the first stage larvae in the experimental group indicated the positive influence of the adaptogen on the embryonic ontogenesis in catfish.

In the course of larvae growth, we have revealed the developmental abnormality, taking into account the number of normally and abnormally developing forms. We introduced the adaptogen into the feed and water in the experimental group.

The number of correctly developing specimen was $89.33 \pm 3.89 \%$. In the control group, the portion of normal forms was $70.0 \pm 2.12 \%$ in larvae obtained from oocytes and embryos untreated with the adaptogen. Primarily, we observed developmental abnormalities of the spine, gills, and eyes. The figure 2 presents the research results.

The survival of larvae is an important indicator of resistance and adaptive flexibility of an organism. It was established that in the presence of adaptogen the larvae survival rate reached $90.67 \pm 2.86$, at the same time in the control group the survival rate was $75.33 \pm 1.78$. The research results are presented in figure 2 .

One of the negative features of catfish is aggressiveness towards the representatives of their species. We got rid of cannibalism in the experimental group, which had got the adaptogen. This negative factor of fish breeding was reduced to $3.33 \pm 1.08 \%$. In the control group without the adaptogen, it reached $18.67 \pm 6.34 \%$.

The increasing viability of embryos in the experimental group and reducing the abnormalities level in the development of catfish embryos we associate with the improvement in biological properties of fertilized eggs after its adaptogen treatment during the swelling period, in cultivation, as well as with the increasing the adaptive flexibility of developing embryos.

We observed the growth and development of catfish larvae at the second research stage. After the yolk sac had dissolved, the catfish larvae switched to exogenous feeding.

During the first week of cultivation, we used artemia nauplius (nauplius Artemia salina) as live starting feed, in a week after switching to exogenous nutrition, we transferred larvae on feeding with the "starting" mixed fodder appropriated to their age and size. Beforehand, all the feeds were enriched with trekrezan.

We added adaptogen in a dose $0.4 \mathrm{~g} / \mathrm{kg}$ of feed. The absolute and average daily biomass gain was studied to assess the effectiveness of adaptogen influence during catfish larval growth.

In the study of weight value, it was found that in 30 days of cultivation in the experimental group the average weight of the larva was $3.90 \pm 0.21 \mathrm{~g}$ and was in 2 times higher than in the control group. Table 1 presents the results of the studies.

Table 1. The effect of the adaptogen on the growth and survival of larvae of the catfish $(M \pm m, n=100)$

\begin{tabular}{|l|c|c|}
\hline \multicolumn{1}{|c|}{ Indicators } & Adaptogen & Control \\
\hline $\begin{array}{l}\text { Average weight of larvae at the } \\
\text { beginning of the experiment, } \mathrm{g}\end{array}$ & $0.05 \pm 0.04$ & $0.05 \pm 0.04$ \\
\hline $\begin{array}{l}\text { Average weight of larvae at the } \\
\text { end of the experiment, g }\end{array}$ & $3.90 \pm 0.21$ & $1.90 \pm 0.20$ \\
\hline Absolute weight gain, g & 3.85 & 1.85 \\
\hline Daily average weight gain, g & 0.13 & 0.06 \\
\hline
\end{tabular}




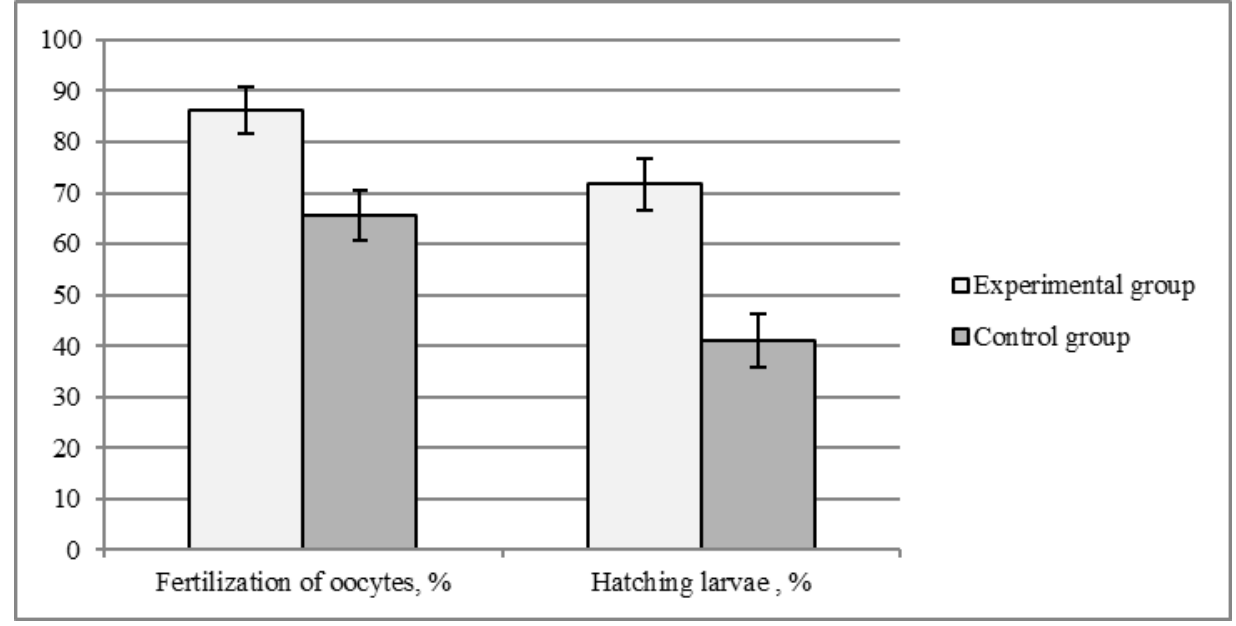

Fig. 1. The influence of adaptogen on the fertilization and the hatching of the first stage larvae

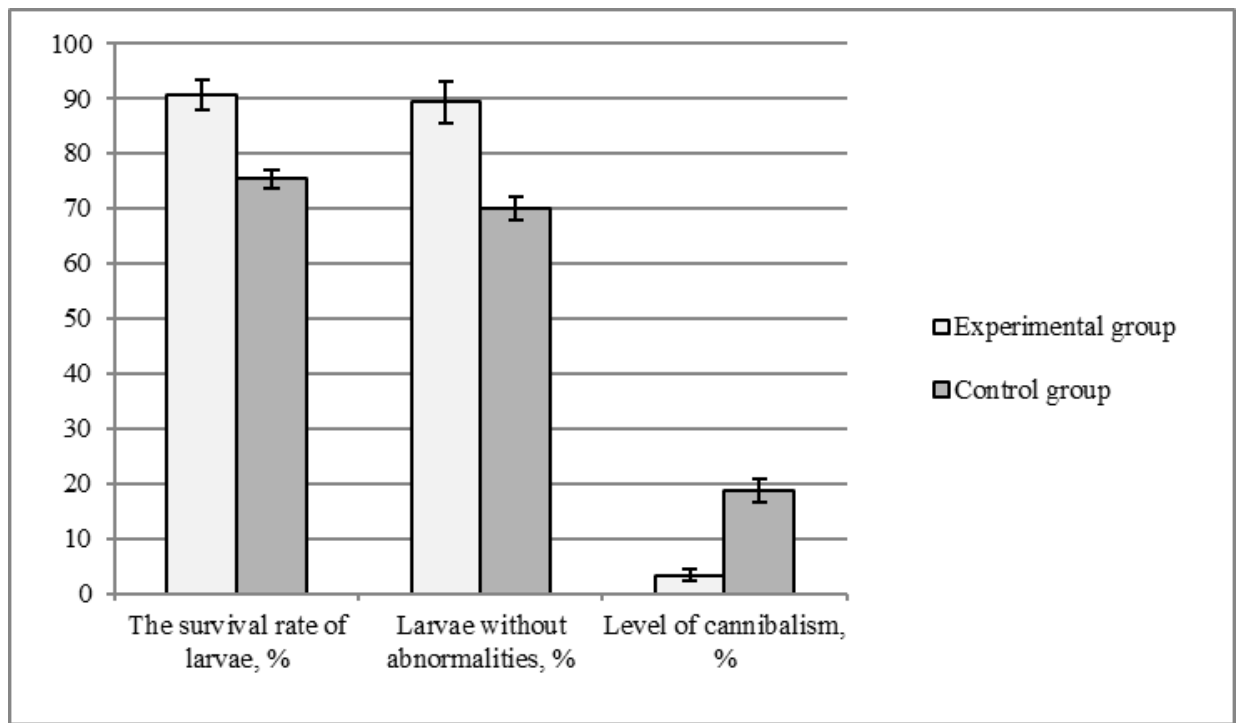

Fig. 2. The survival rate, the level of abnormalities and cannibalism in larvae of the catfish in the presence of the adaptogen

\section{Discussion}

A negative factor in modern fish farming is the high fish mortality at the early stages of ontogenesis. The cause of fish death is often the chronic stress that is unavoidable in the conditions of artificial breeding. During this state the concentration of steroid hormones in the organism decreases, it retards the gonads maturation and reduces the quality of sexual fish products. According to statistics at the hatcheries in Russia, 70-80\% of unfertilized eggs have the deformation of eggs and their shells, a tendency to reduce the size of eggs because of the decrease of the protein amount in a single egg. We have discovered the developmental abnormalities in the olfactory organs, disorders in the eyes structure; reduction or underdevelopment of the opercle, the barbels, the digestive tract, etc. at $50 \%$ of fries.

Embryonic and larval stages in the life cycle of fish are the most vulnerable phases. The highest mortality rate is noted namely at the stages of fertilization, embryonic and larval ontogenesis.

The search for factors of decreasing the mortality rate at the early stages of ontogenesis in artificial breeding made us turn to the theory of adaptogens and the phenomenon of "the state of nonspecific increased resistance" that occurs in the organism under the adaptogen's influence.

The adaptogen trekresan used in our experiments is a synthetic analog of natural ginseng, and it demonstrated good results during embryo cultivation, increased larvae hatching, larvae survival rate and decreased the level of developmental abnormalities and cannibalism inherent to catfish larvae. Studies have shown that the use of adaptogen had a positive influence on the growth and development of larvae. For 30 days of the experiment in growing larvae with trekrezan, they demonstrated the biomass gain two times faster than in the control group.

Despite all their effective properties, fish breeders have never used adaptogens until now. Our results are new and allow us to recommend the use of adaptogens at 
the early stages of ontogenesis in industrial aquaculture of catfish.

\section{Conclusion}

The adaptogen trekrezan use had a positive effect on the process of the embryo culture: the hatching of the first stage larvae increased by $30.67 \%$, larvae survival by $15.34 \%$, the level of developmental abnormalities decreased by $19.33 \%$, and the level of cannibalism decreased on average by $15.34 \%$.

The use of trekrezan had a positive influence on the growth and development of larvae. The absolute and average daily gain was two times higher during catfish larval growth in the conditions with adaptogen and with the use of feed enriched with trekrezan than in larvae breeding without any adaptogen.

\section{References}

1. R. Rui, N.M. Bandarra, M.L. Nunes, Int. J. of Food Sci. and Technol. 42, 3 (2007)
2. M.A. Suleiman, R.J. Solomon, Direct Res. J. Vet. Med. Anim. Sci. 2, 3 (2017)

3. V.T. Okomoda, Chu Koh I. Chong, S. Md. Shahreza, Aquacult. Res. (2017)

4. N. Gadisa et al., Int. J. of Fisheries and Aquatic Studies 5, 2 (2017)

5. E. Romanova, E. Fedorova, E. Kamaletdinova, Zootechnics 10 (2014)

6. E. Romanova, V. Lyubomirova, V. Romanov, M. Mukhitova, T. Shlenkina, The Egyptian J. of Aquatic Res. 44, 4 (December 2018)

7. E. Ivanišová, M. Kačániová, J. Petrová, R. Staňková, L. Godočíková, Scientific Papers Animal Science and Biotechnologies, 49(1), 96-102 (2016)

8. A. Panossian, Annals of the New York Acad. of sci. 1401, 1 (2017)

9. I. Lunegova, A. Kuznetsov, A. Lunegov, Bulgarian J. of Veter. Med. 20, S1 (2017)

10. J. Grünwald, H. Stier, S. Bruhn, B. Goyvaerts, Zeitschrift fur Phytotherapie: offizielles Organ der Ges. f. Phytotherapie 32, 4 (2011) 